\title{
Research on q-p-shaped elevated traffic system in wetland and forest parks
}

\author{
Ning Kang*, Yan Liu, and Qi Zhang \\ School of Art, Hubei Polytechnic University, Huangshi, 435003, China
}

\begin{abstract}
Ecological protection is the basis of the construction of wetland and forest parks and therefore any development and utilization should take it as the premise. The traffic system planning and positioning of wetland and forest parks should also follow this principle. The establishment of the q-p-shaped elevated traffic system equipped with ring elevated passage and local installation of ground and over-ground viewing platforms in whole park can achieve the diversion of commuter cars, bicycles and walking tourists which is conducive to solving the problems of patch fragmentation, human activities interference and single viewing form in wetland construction. In this paper, the ways of creating q-p-shaped elevated traffic system is introduced in detail and this study can provide reference for the planning and design of wetland and forest parks.
\end{abstract}

\section{Features of wetland and forest parks}

Wetland, forest and ocean are known as the world's three major ecosystems. Wetland park and forest park or wetland forest mixed park, as an effective way to solve the contradiction between wetland forest protection and development, as well as the most important form and carrier of eco-tourism have the function of adjusting local climate and improving urban heat island effect, which can effectively improve the adaptability of cities under the background of global change. As a new type of tourist destination, it can also reduce the pressure of nature reserves, scenic spots and other tourist destinations, and promote the sustainable development of the city in return.

At the same time, wetland and forest are vulnerable to human disturbance. Therefore, the contradiction of maintaining original habitat of wetland and forest will become an important base for ecosystem research in ecologically fragile areas.

\section{Analysis of traffic particularity and problems in wetland and forest parks}

Wetland and forest park are different from ordinary park. The basic basis of planning and construction is ecological restoration and protection. Therefore, the transportation planning of wetland park should be based on ecological protection, guided by wetland and forest experience and education, and at the same time taking into account recreational activities.

\subsection{Habitat patches fragmentation}

If the wetland and forest parks laying the traffic road on the ground like other ordinary parks, it will lead to the fragmentation of the forest habitat wetland habitat patches and seriously affect the integrity of the native plant community. At the same time, the migration and reproduction of animal and plant activities will also be affected to some extent. All these are contrary to the development of wetland should focus on the concept of ecological protection.

\subsection{Uncontrollability of visitors' activities}

There are many blind areas to monitor and control, and it is difficult to manage them if the traffic roads are laid on the ground allowing the visitors go and stop at will to play. It is easy to appear trampling and climbing plant plants, or other uncivilized behaviours such as capturing animals in the park, causing losses to the park and the ecological environment. In addition, the wetland and forest environment have its own particularity. It is easy for tourists to get lost, enter the mire or encounter dangerous creatures when they traveling at will.

\subsection{Singleness of viewing line of landscape in height}

When tourists in the ground traffic, their viewing line of sight are about $1.2 \mathrm{~m}-2.0 \mathrm{~m}$, while that of Asians is about $1.5 \mathrm{~m}$. Therefore, in the wetland forest environment, this height for large and medium-sized plants can only look at the trunk, while the branches and leaves can only be seen by looking up. And tourists are unable to appreciate the whole picture.

\footnotetext{
* Corresponding author: kangning@hbpu.edu.cn
} 


\section{Design of $q-p-s h a p e d$ elevated traffic system}

\subsection{Layout of $q-p-s h a p e d$ elevated traffic system}

\subsubsection{Plane form of layout}

Elevated traffic network adopts q-p-shaped plane layout. A glimpse and stroll travelling are combined to form a ring around the whole park. The O-shaped viewing platforms with diameter of $25 \mathrm{~m}$ shown in figure 1 are set up in high value places, service facilities, open view places, scenic spots and unique scenery locations.

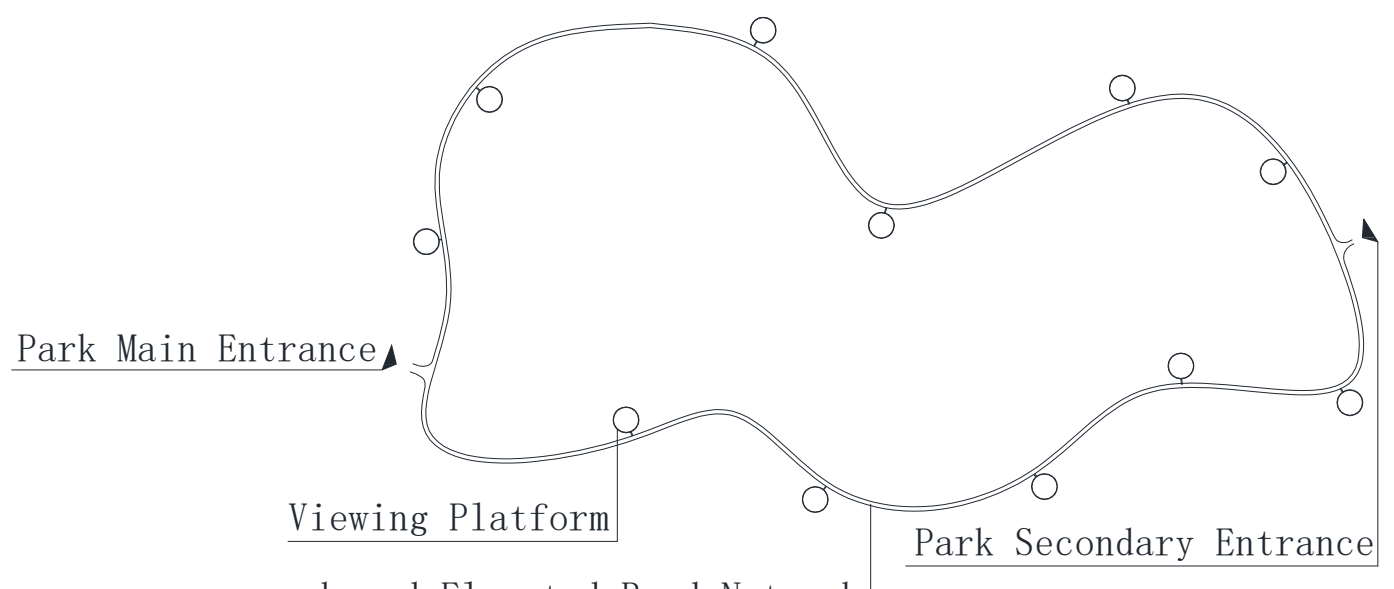

q-p shaped Elevated Road Network

Fig.1. Plane form of q-p-shaped elevated traffic system

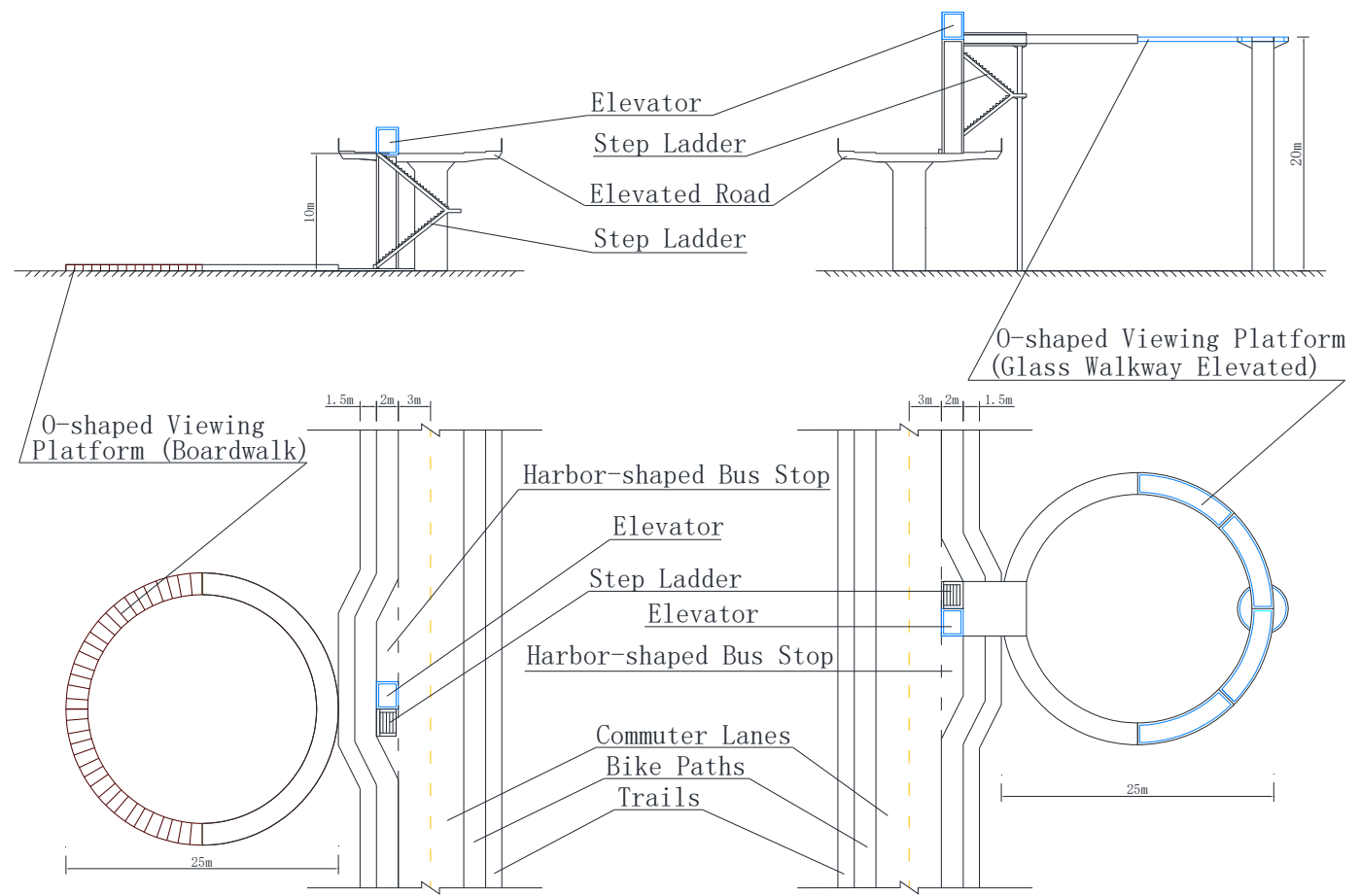

Fig.2. Stereo-plane form of q-p-shaped elevated traffic system

\subsubsection{Stereo-plane form of layout}

The stereo-plane form of elevated traffic network adopts the structural design of monolithic single floor (not less than $10 \mathrm{~m}$ high) and partial (viewing platforms) two layers (lower to the ground or not less than $20 \mathrm{~m}$ high). Setting up O-shaped ground viewing platforms (combined with wooden trestle road) at the service 
facilities and unique scenery sites with high-value sightseeing so that it is convenient for tourists to enjoy leisure and recreation, supply and rest, participate in experience and other functional needs. The height not lower than $20 \mathrm{~m}$ of O-shaped viewing platforms (combined with glass trestle road) are set up at open view places, beautiful scenery and unique scenery locations, which is convenient for tourists to experience novelty, ascend a height to enjoy a distant view and stand and watch. The two floors are connected by sightseeing elevators and steps for the convenience of special groups and ordinary tourists.

\subsubsection{Ways of traffic diversion}

Park commuter lanes were set up two-way two-lane with width of $6 \mathrm{~m}$ and the maximum speed limit of $20 \mathrm{~km}$ per hour. Commuter buses start on time for fast trips and special groups of tourists. And stops are set up at each viewing platform to facilitate visitors to get off and enter the $\mathrm{O}$-shaped viewing platform for sightseeing.

As shown in figure 2, bike lanes, secondary park road, are provided for cycling enthusiasts with two-way lanes, which are located on both sides of the commuter lane and $2 \mathrm{~m}$ in width in one direction. Walking paths for slow tour and hiking enthusiasts, three-level garden road, are located on both sides of the bike path with the width of $1.5 \mathrm{~m}$.

\subsection{Viewing platform sites selection}

Fully respecting the natural sites and minimizing the traces of man-made alterations. The park landscape resources can be excavated in depth through landscape evaluation technology, and the scenic spots with wide view, beautiful scenery, unique places with high value for play and service facilities can be selected for visitors to get off the bus and enjoy a slow tour.

\subsubsection{Open fields of vision}

Parking sites should be set up in locations with broad views in the park, such as depending on the terrain, skilfully taking advantage of mountain peaks, and setting viewing platforms with a height of no less than $20 \mathrm{~m}$ (combined with glass-paved trestles). Emphasis should be placed on the construction of the permeability of landscape viewing corridors, so as to ensure the open space with broad vision and meet the needs of tourists for sensory stimulation and distant viewing.

\subsubsection{Beautiful scenery and unique landscape}

In addition, parking sites are also set up in unique scenic spots, such as typical plant communities in typical terrain and physiognomy areas representing wetland forest characteristics. At the same time, viewing platforms with a height of not less than $20 \mathrm{~m}$ (combined with glass laying plank road) or viewing platforms on the ground (combined with antiseptic wooden laying plank road) with bench seat are set up, so that visitors can experience the mysterious nature of nature, appreciate the seasonal and ecological changes of the plant landscape, and satisfy the functional needs of tourists' novelty experience and enjoying the rest.

\subsubsection{High value for sightseeing}

In places with high value for sightseeing, such as developed water area, wooden trestle road viewing platform can be set up on the ground. Hydrophilic platform for bird watching platforms can be designed in suitable areas to meet the functional requirements of tourists' boat feeding fish, bird watching and lotus watching. It makes the visitors intuitively feel the characteristics of birds and aquatic organisms' activities, combined with commentary boards and landscape sculptures, so that tourists can deepen their understanding of local ecology while visiting and sightseeing and therefore access to popular science education in ecology and closer to the nature.

\subsubsection{Location of service facilities centralized}

In places with service facilities, such as snack shop, toilet, exhibition hall, tea house, fishing area and picking area, an observation platform connected with the service facilities can be set up on the ground to meet the needs of tourists for entertainment, science, leisure and other functions, providing tourists with opportunities to get close to nature, enhancing the sense of participation and reflecting the charm of the park.

\subsection{Superiority of q-p-shaped elevated traffic system}

\subsubsection{Maximum protection of the integrity of the ecological environment}

The biggest advantage of the double q-p-shaped elevated transportation system is to maximize the protection of wetland native plant communities, protect animal habitats, and build the integrity of the original ecosystem with a strong sense of hierarchy, rich biodiversity and stable and orderly structure when developing and utilizing the resources.

\subsubsection{The scope and mode of activities of tourists are controllable in the whole process}

In addition, the scope and mode of activities of tourists can be controlled in the whole process. To guarantee the safety of tourists' lives and property, In the park, people and vehicles are diverted to ensure that the park's animals and plants are not disturbed by human activities, and that the scope and mode of activities of tourists can be controlled in the whole process, so as to ensure the safety of tourists 'lives and property. Besides this, only each commuter car parking station in the park needs to invest supervision and service manpower, which can also reduce the expenditure of the park. 


\subsubsection{New viewing experience}

The viewing platform with a height of $20 \mathrm{~m}$ can change the traditional viewing angle of the massive landscape and the viewing angle of the overlook. It will take visitors a different viewing experience by the way of new viewing height. And meanwhile partial deep into the forest of the ground observation platform allows visitors to experience the mystery of nature.

\section{Conclusions}

The traffic system planning of wetland parks should be based on ecological protection, guided by wetland and forest experiencing and education, and meanwhile taking leisure and recreational activities into account. The q-pshaped elevated traffic system is the whole park ring elevated traffic, which is the traffic system with ground and over ground double-deck viewing platform designed at places with open view, beautiful scenery, unique scenery, high value of play and service facilities.

This system can not only protect the integrity of the ecological environment to the greatest extent, but also bring different sensory experience to tourists. Besides, it can also assist park supervision services and improve the traffic organization and operation efficiency of the park. The study of q-p-shaped elevated traffic system in this paper has certain reference and guidance value in the traffic planning and construction of wetland and forest parks.

\section{Acknowledgement}

This work is funded by Opening fund of the research centre for mining and metallurgy culture and economic and social development in the middle reaches of Yangtze river, Hubei Province (No.2016kywh12).

\section{References}

1. Zhai Y, Baran P K, Wu C. Spatial distributions and use patterns of user groups in urban forest parks: An examination utilizing GPS tracker[J]. Urban forestry \& urban greening, 2018, 35: 32-44.

2. $\mathrm{Li} \mathrm{T}$, Gao X. Ecosystem services valuation of lakeside wetland park beside Chaohu Lake in China[J]. Water, 2016, 8(7): 301.

3. Li Y, Nani L I. Progress and Prospect of Studies on Landscape Health of Urban Wetland Park[J]. Journal of Landscape Research, 2016, 8(4): 63.

4. Grošelj P, Hodges D G, Stirn L Z. Participatory and multi-criteria analysis for forest (ecosystem) management: A case study of Pohorje, Slovenia[J]. Forest Policy and Economics, 2016, 71: 80-86.

5. Pueyo-Ros J, Ribas A, Fraguell R M. A cultural approach to wetlands restoration to assess its public acceptance[J]. Restoration Ecology, 2019, 27(3): 626-637.

\section{Biography}

Ning Kang (corresponding author), female, lecturer, research interests: environmental design, urban planning and design. Email: kangning@hbpu.edu.cn

Yan Liu, female, professor, research interests: artistic design. Email: 121100926@qq.com

Qi Zhang, female, lecturer, research interests: environmental design. Email: 312324635 @qq.com 